\title{
AIMCO CRITERIA (ADEQUACY OF INVASIVE MANAGEMENT OF ACUTE CORONARY SYNDROME IN OLDER PATIENTS): A PROPOSAL TO ADAPT DECISION MAKING
}

\author{
B. Alvarez-Fernandez ${ }^{1}$, I. Ribera Cabeo ${ }^{2}$, M.R. Bernal-Lopez ${ }^{2,3}$, M. de Mora Martin ${ }^{4}$, C. Urbano Carrillo ${ }^{4}$, \\ G. Quesada Garcia ${ }^{5}$ J.A. Arboleda ${ }^{5}$ R. Gomez Huelgas²
}

Dear Editor,

The invasive treatment is the recomemded therapeutic approach by the guidelines in patients with non-STelevation acute coronary syndrome (NSTE-ACS) and high level of risk of reinfarction or death $(1,2)$. Recommendations based on randomized trials and observational studies that have found strong evidence that invasive treatment is more beneficial in elderly patients with NSTE-ACS than younger subjects, even considering the increased risk of bleeding in the elderly. Nonetheless, our data (3) and in other studies of neighbouring countries $(4,5)$ show a tendency to perform invasive treatment of NSTE-ACS in patients with a lower risk and a younger age, depriving many patients of invasive treatment solely because of their age without any associated functional or cognitive disorders to warrant this.

Decision-making in older patients is a complex process that should be based on a comprehensive assessment of the patient as a tool to weigh the risks and benefits of treatment. Specific risk scores do not include elements that are essential for the assessment of elderly patients with heart disease. In many cases comorbidity, functional capacity and cognitive status are more determinant of patient survival and quality of life than the actual process to be treated. All this, together with the patient's wishes, should form the framework supporting the complex development of therapeutic decisions.

Hence we proposed to reach agreement among professionals responsible for the care of these patients

1. Geriatric Unit- Internal Medicine Department, Regional University Hospital of Málaga (Carlos Haya Hospital), Spain; 2. Internal Medicine Departament, Biomedical Institute of Málaga (IBIMA) Regional University Hospital of Málaga (Carlos Haya Hospital), Spain; 3. Ciber Fisiopatología de la Obesidad y Nutrición. Instituto de Salud Carlos III. Madrid, Spain; 4. Cardiology Department, Biomedical Institute of Malaga (IBIMA), Regional University Hospital of Málaga (Carlos Haya Hospital), Spain; 5. Critical Care and Emergency Department, Regional University Hospital of Málaga (Carlos Haya Hospital), Spain.

Corresponding Author: M Rosa Bernal-Lopez. Internal Medicine Department, Biomedical Research Institute of Malaga (IBIMA), Regional University Hospital of Malaga (Carlos Haya Hospital), Avda. Hospital Civil, s/n. 29009. Malaga, Spain. Phone: +34 951290346; Fax: +34 951290302. E-mail address: robelopajiju@yahoo. es; B Alvarez-Fernández: Geriatric Unit-Internal Medicine Department, Regional University Hospital of Malaga (Carlos Haya Hospital), Avda. Hospital Civil, s/n. 29009. Malaga, Spain. balvarez@t2v.com (cardiologists, geriatricians, internists, specialists in emergency and critical care) on criteria that could enable the correct identification of the limitations of invasive management. we designed a qualitative study. A multidisciplinary committee (MC) was formed, composed of five experts (cardiologist, geriatrician, intensivist, internist and emergency physician), who defined and weighted the factors that can lead to improper use of invasive procedures in elderly patients with NSTE-ACS due to the high risk of bleeding, the presence of geriatric syndromes and/or significant comorbidities that limit life expectancy and/or quality of life. These factors were discussed in joint sessions and separately in the three clinical departments involved (Cardiology, Internal Medicine, Critical Care and Emergency), modifying them as a result of the arguments presented in these sessions. Subsequently, the agreed criteria were then sent in a personal letter to each member of the departments involved so that they could examine the criteria in detail and make any pertinent observations. The MC then reviewed the comments submitted by the various professionals and made any modifications considered appropriate. Consensus criteria are shown as "AIMCO criteria" (table 1)

These criteria are intended to be a user-friendly application that will help reduce the variability in clinical practice of the different medical specialists who treat elderly patients with NSTE-ACS, as well as facilitate clinical judgment based on reasonable criteria to assess whether carrying out invasive treatment is not in fact indicated to improve the current or future prospects of the patient in terms of survival and/or quality of life. This is not a score of risk to determine the risk of an invasive treatment, only a consensus regarding in which cases it is justified not to perform invasive treatment in elderly patients with NSTE-ACS despite such treatment being indicated.

The AIMCO criteria represent the first proposal to adapt indications for invasive treatment in elderly patients with NSTE-ACS. With these consensus criteria we sought specificity over sensitivity, i.e., we considered it more important that the excluded patients 
Table 1

AIMCO Criteria (Adequacy of Invasive Management of Acute Coronary Syndrome in Older Patients)

NOTE: YOU MUST CONSIDER THE PATIENT'S WISHES

Points

History of major bleeding in the past 3 months (hemorrhagic stroke, gastrointestinal hemorrhage, retinal hemorrhage) [1 point]

Severe thrombocytopenia or any serious disorder of hemostasis. [1 point]

Previous organ failure moderate [1 point] / severe [2 points]. Excluding heart failure.

Previous severe heart failure [2 points]

Significant systemic disease (oncologic disease with no prospect of cure, advanced amyotrophic lateral sclerosis (ALS) or other diseases with expected survival $<1$ year) [2 points]

Creatinine clearance in the acute stage $(\mathrm{MDRD})<30 \mathrm{~mL} / \mathrm{min}[1$ point $]$

Moderate to severe pre-dependency ( $<55$ points on Barthel scale) [1 point] (Moderate: needs some help for some basic activities such as toileting, dressing, walking, climbing stairs, occasional incontinence, eating, using the toilet ...)

Prior moderate cognitive impairment (difficulty choosing clothes or remembering names of grandchildren, cannot live alone due to their cognitive impairment, cannot handle money, but roams the house without help, gets up from the bed alone, and maintains language that allows communication) [1 point], severe (dependent for bathing, eating, toileting, serious communication gap...) [2 points]

\section{TOTAL POINTS}

- A score of 2 on a single item, or a total of 4 points or more on different items, would indicate that invasive management may have a high immediate risk or be inadequate to improve future perspectives regarding patient survival and/or quality of life.

CLARIFICATIONS:

- Severe thrombocytopenia: platelet count $<50,000 / \mathrm{mm} 3$

ORGAN FAILURE:

- Moderate renal impairment: prior creatinine clearance between 59-30 $\mathrm{ml} / \mathrm{min}$

- Severe renal impairment: prior creatinine clearance $<30 \mathrm{ml} / \mathrm{min}$.

- Moderate hepatic impairment: cirrhosis with minimal complications (mild pancytopenia, ascites controlled with drugs, has not had episodes of encephalopathy, bilirubin $<3$, INR $>1.5$ because of the liver)

- Severe hepatic impairment: cirrhosis with complications more important than severe cirrhosis

- Chronic obstructive pulmonary disease (COPD). Moderate: treatment with beta 2-agonists, anticholinergics ++ inhaled corticosteroids regularly, with habitual dyspnea to walking pace without oxygen.

- Severe COPD: chronic home oxygen with significant functional limitations secondary to pulmonary disease.

- Previous severe heart failure: common functional class III / IV, previous persistent edema despite adequate treatment, readmissions for this cause in the last 6 months, ejection fraction $<30$

- If there is more than one organ failure in the same patient, only the most severe stage organ failure will be marked. In any event, this will only score one point

- IMPORTANT SYSTEMIC DISEASE. Refers to diseases which themselves limit survival and/or quality of life: advanced cancer, amyotrophic lateral sclerosis (ALS). Excluded from this section are: organ failure from the previous section and heart failure.

had inadequacy criteria than that some patients without exclusion criteria were subsequently excluded because technical or anatomical features or clinical events ruled out the invasive procedure. We are confident that the application of the AIMCO criteria will serve as a new communication tool between the different specialists involved in the care of these patients and be an important feature in complex decision-making in the elderly. The usefulness of these criteria should be tested in future studies (Phase 2) to evaluate whether their application helps to reduce variability in clinical practice and to improve clinical outcomes in the elderly population, especially in very elderly individuals (over 75 years old).
Conflict of interest:The authors have no conflicts of interest to disclose

\section{References}

1. Amsterdam EA, Wenger NK, Brindis RG, Casey DE Jr, Ganiats TG, Holmes DR Jr, Jaffe AS, Jneid H, Kelly RF, Kontos MC, Levine GN, Liebson PR, Mukherjee D, Peterson ED, Sabatine MS, Smalling RW, Zieman SJ; ACC/ AHA Task Force Members; Society for Cardiovascular Angiography and Interventions and the Society of Thoracic Surgeons. 2014 AHA/ ACC guideline for the management of patients with non-ST-elevation acute coronary syndromes: executive summary: a report of the American College of Cardiology/American Heart Association Task Force on Practice Guidelines. Circulation. 2014. 23;130(25):2354-94

2. Authors/Task Force Members, Roffi M, Patrono C, Collet JP, Mueller C, Valgimigli M, Andreotti F, Bax JJ, Borger MA, Brotons C, Chew DP, Gencer B, Hasenfuss G, Kjeldsen K, Lancellotti P, Landmesser U, Mehilli J, Mukherjee D, Storey RF, Windecker S. 2015 ESC guidelines for the 
management of acute coronary syndromes in patients presenting without persistent ST-segment elevation. Eur Heart J. Aug 2015 29. pii: ehv320.

3. Alvarez-Fernandez B, Bernal-Lopez MR, Cabeo Inmaculada R, Martin Manuel DM, Carrillo Cristobal U, et al. Elderly Patients with NonSTElevation Acute Coronary Syndrome: A Proposal to Adapt Decision Making. J Gerontol Geriat Res 2015. 4:227. doi:10.4172/2167-7182.1000227

4. Steg PG, Kerner A, Van de Werf F, MD, López-Sendón J, Gore JM, FitzGerald G, Feldman LJ, Anderson FA, Avezum A, MD; for the Global Registry of Acute Coronary Events (GRACE) Investigators Impact of In Hospital Revascularization on Survival in Patients with Non-ST-Elevation Acute
Coronary Syndrome and Congestive Heart Failure. Circulation. 2008. 118: $1163-1171$

5. Bhatt DL, Roe MT, Peterson ED, Li Y, Chen AY for the CRUSADE Investigators. Utilization of Early Invasive Management Strategies for HighRisk Patients With Non-ST-Segment Elevation Acute Coronary Syndromes Results From the CRUSADE Quality Improvement Initiative. JAMA 2004. 292:2096-2104 